\title{
Extracranial Neuroblastoma
}

National Cancer Institute

\section{Source}

National Cancer Institute. Extracranial Neuroblastoma. NCI Thesaurus. Code C5437.

A neuroblastoma arising from an anatomic site other than the brain. 